\title{
Ginkgo biloba extract promoted the astrocyte-mediated clearance of intercellular alpha-Syn via autophagy and proteasome pathway
}

\author{
Yi Fan, Jun Hua, Nuo Yin, Gang Hu \\ Department of Pharmacology, Nanjing Medical University, China
}

Background: The accumulation of aggregated forms of the alpha-Synuclein (alpha-Syn) is associated with the pathogenesis of Parkinson's disease (PD). Increasing evidences have shown the protective effects of Ginkgo biloba extract (GBE) on motor activity after PD. Astrocyte, a key cell type in the central nervous system (CNS), plays an important role in PD process. However, whether astrocytes contribute to alpha-Syn elimination, and ginkgolides exert neuroprotection against alpha-Syn aggradation are unclear, and the underlying molecular mechanisms remain unidentified.

Methods: In the present study, we investigated whether astrocytes might engulf and degrade recombinant alpha-Syn monomers and aggregates, and the underline mechanisms in vitro. Then, we evaluated the effects of ginkgolide B (GB) and bilobalide $(\mathrm{BB})$ on astrocytic clearance of alpha-Syn in vitro, and the alpha-Syn content in A53T mutant alpha-Syn transgenic mice.

Results: We showed that astrocytes engulfed and degraded extracellular aggregated recombinant human alpha-Syn by Western blotting and immunocytochemistry assay. Meanwhile, we observed that the clearance of alpha-Syn by astrocytes was partially abolished by proteasome inhibitor MG132 or/and autophagy inhibitor 3-Methyladenine (3-MA). We further found that Ginkgo biloba active ingredients $\mathrm{GB}$ and $\mathrm{BB}$ reduced intracellular alpha-Syn by Western blotting and immunocytochemistry assay. Furthermore, MG132 and 3-MA reversed the GB and BB induced decrease of intracellular alpha-Syn. More importantly, we found that GB and BB treatment reduced indirect neurotoxicity to neurons induced by alpha-Syn-stimulated astrocytic conditioned medium.

Conclusions: We firstly find that astrocytes engulf and degrade alpha-Syn aggregates via proteasome and autophagy, and GB and BB enhance astrocyitc clearance of alpha-Syn partially by promoting cell autophagy, which gives us an insight into the novel therapy for PD in future. 